\title{
ON THE MODELING OF THE TRANSPORT OF PARTICLES IN TURBULENT FLOWS
}

\author{
ThIERry Goudon ${ }^{1}$ ANd FrÉdÉRIC POUPAud ${ }^{2}$
}

\begin{abstract}
We investigate different asymptotic regimes for Vlasov equations modeling the evolution of a cloud of particles in a turbulent flow. In one case we obtain a convection or a convection-diffusion effective equation on the concentration of particles. In the second case, the effective model relies on a Vlasov-Fokker-Planck equation.
\end{abstract}

Mathematics Subject Classification. 35B25, 35Q99.

Received: September 25, 2003.

\section{INTRODUCTION}

In this paper, we investigate models describing the evolution of particles dispersed in a turbulent fluid. These models arise for instance in combustion theory, as in the description of injection processes in Diesel engines, or rocket propulsors. The particles are described through a density function $f(t, x, v) \geq 0$. Given $\Omega \subset \mathbb{R}^{N}$ and $\mathcal{V} \subset \mathbb{R}^{N}$, the quantity

$$
\int_{\Omega} \int_{\mathcal{V}} f(t, x, v) \mathrm{d} v \mathrm{~d} x
$$

is interpreted as the number of particles occupying at time $t \geq 0$, a position $x$ in the set $\Omega$, and having their velocity $v$ in the set $\mathcal{V}$. The fluid is described through its velocity field $u(t, x) \in \mathbb{R}^{N}$. Forces exerted on the particles are supposed to reduce to the friction forces exerted by the surrounding fluid. They are supposed to be proportional to the relative velocity $(u-v)$ : the so-called Stokes force is then defined by

$$
M F(t, x)=6 \pi \mu a(u(t, x)-v)
$$

where:

- $\mu$ is the dynamic viscosity of the fluid;

- $a$ is the radius, supposed constant, of the particles;

- $M$ is the mass of a particle. In the sequel, we assume that the particles are spherically shaped, so that $M=\frac{4}{3} \pi a^{3} \rho_{p}, \rho_{p}$ being the mass density of the particles.

\footnotetext{
Keywords and phrases. Fluid-particles interaction, hydrodynamic limits, turbulence effects.

1 Laboratoire Paul Painlevé U.M.R. 8524, CNRS, Université des Sciences et Technologies de Lille, Cité Scientifique, 59655 Villeneuve d'Ascq Cedex, France. e-mail: thierry.goudon@math.univ-lille1.fr

2 Laboratoire J.A. Dieudonné U.M.R. 6621, CNRS, Université Nice-Sophia Antipolis, Parc Valrose, 06108 Nice Cedex 2, France. e-mail: poupaud@math.unice.fr 
Next, we shall suppose that any collision effects can be neglected. As pointed out to us by the referee, note that the high dilution of the particles (typically the volume fraction occupied by the particle is less than 0.01) is not enough to guaranty the validity of this assumption. It also relies on the interaction forces between the particles. We refer to this aspect to the deep comments in the review paper of Villani [25]. Therefore, the evolution of the density $f$ is governed by the following Vlasov-type equation

$$
\partial_{t} f+\nabla_{x} \cdot(v f)+\nabla_{v} \cdot\left(\frac{9 \mu}{2 a^{2} \rho_{p}}(u-v) f\right)=0
$$

Considering a cloud of $P$ particles, the trajectories $\left(X_{i}(t), V_{i}(t)\right), i \in\{1, \ldots P\}$, followed by the particles are defined by the ODE system

$$
\frac{\mathrm{d}}{\mathrm{d} t} X=V, \quad \frac{\mathrm{d}}{\mathrm{d} t} V=F(t, X, V)=\frac{9 \mu}{2 a^{2} \rho_{p}}(u(t, X)-V) .
$$

Hence, the quantity

$$
f(t, x, v)=\sum_{i=1}^{P} \delta\left(x=X_{i}\right) \otimes \delta\left(v=V_{i}\right)
$$

obeys (1).

A complete modeling of the fluid/particles evolution involves a coupled equation governing the evolution of the velocity field $u$. We refer for instance to the modeling presented by Russo-Smerecka [24], Herrero-Lucquin-Perthame [15], or Jabin-Perthame [18] when considering potential flows, and, more recently, the work of Gavrilyuck-Teshukhov [9]. A coupling involving the incompressible Navier-Stokes equation, with a force term defined by moments of $f$ is described by Caflisch-Papanicolaou [4]. More details on the physical background can be found, e.g. in the treatises of Brock-Hidy [3], or Williams [26] and the article of O'Rourke [22]. We also refer to the note of Desvillettes [6] and the references therein. Mathematical treatments of these models are due to Hamdache [14], and Jabin [16].

In this work, we shall assume that the velocity field is given: since there are neither collisions between particles, nor influence of the particles on the fluid evolution, the situation is said to correspond to a "very thin spray". We suppose the velocity field can be split into a mean part $u_{m}$ and a fluctuating part $u_{f l}$. We are interested in asymptotic regimes where the scales of the fluctuations of the velocity field interact with the typical scales of the evolution of the density $f$. Such kind of question is reminiscent to hydrodynamic limits, see Golse [10], and for application to such fluid/particles interactions model we mention the works of Berthonnaud [1], Domelevo-Vignal [7], Goudon [11], Jabin [17], and Goudon-Jabin-Vasseur [12, 13]. A part of the questions we address here are dealt with, by using a different approach, by Clouet-Domelevo [5] and Domelevo-Villedieu [8]. We also quote for a derivation from a physical viewpoint the work of Zaichik [27]. Here, we shall use the method introduced by Poupaud-Vasseur [23] which relies on the properties of decorrelation in time of the random fluctuation field. This method has also been applied to deal with general linear hyperbolic systems by Brassart [2] or to investigate plasma turbulence in tokamaks by Loeper-Vasseur [21].

The paper is organized as follows. In Section 2, we write equation (1) in dimensionless form. The different regimes are determined by physical assumptions and a convenient choice of units. In Section 3, we detail precisely the mathematical assumptions that we use and we give the statements of the main results obtained in the paper. Then, Section 4 is devoted to the proofs. 


\section{Dimensionless equations}

\subsection{Dimension analysis of the Vlasov equation}

First of all, we remark that the coefficient associated to the Stokes force in (1) is homogeneous to the inverse of a time. We denote this relaxation time by

$$
\mathcal{T}_{\text {rel }}=\frac{2 a^{2} \rho_{p}}{9 \mu}
$$

(This quantity is often referred to as the Stokes settling time.) We shall now discuss other physical quantities which appear within the model.

\subsubsection{The turbulent velocity field}

Here, turbulence is modeled through the randomness of the fluctuation part of the velocity field $u_{f l}$. For the sake of simplicity, we assume that the mean velocity vanishes $u_{m}=0$, and we simply denote $u=u_{f l}$. A key assumption is that $u_{f l}(t, x)$ and $u_{f l}(s, y)$ are correlated only on a short interval of time; precisely when $|t-s| \leq \mathcal{T}_{\text {cor }}$. Hence, the correlation time $\mathcal{T}_{\text {cor }}$ has to be compared to the relaxation time $\mathcal{T}_{\text {rel }}$. Finally, fluctuations of $u_{f l}$ occur in space with a characteristic length $\mathcal{L}_{f l}$. Precisely, we have

$$
u_{f l}(t, x)=\mathcal{U}_{f l} U\left(t / \mathcal{T}_{c o r}, x / \mathcal{L}_{f l}\right)
$$

where $U$ is a dimensionless random field and $\mathcal{U}_{f l}$ stands for the amplitude of the fluctuations. We will make more precise the mathematical assumptions on $U$ later on. Let us finally define the correlation length $\mathcal{L}_{\text {cor }}=\mathcal{U}_{f l} \mathcal{T}_{\text {cor }}$. It corresponds to the characteristic length of a path of a particle having velocity $\mathcal{U}_{f l}$ on a time interval $\left(t, t+\mathcal{T}_{\text {cor }}\right)$.

\subsubsection{Dimensionless parameters}

Up to now we have determined four physical constants which characterize the model. We are dealing with two time scales:

$$
\begin{cases}\mathcal{T}_{\text {rel }} & \text { the relaxation time associated to the Stokes force, } \\ \mathcal{T}_{\text {cor }} & \text { the time on which velocity fields remain correlated, }\end{cases}
$$

and two length scales:

$$
\begin{cases}\mathcal{L}_{f l} & \text { the typical length of the fluctuations of the velocity field, } \\ \mathcal{L}_{c o r} & \text { the correlation length of the trajectories of the velocity field. }\end{cases}
$$

In order to write equation (1) in a dimensionless form, we need to introduce a time unit $\mathrm{T}$, a length unit $\mathrm{L}$ and a velocity unit $\mathrm{V}$. Throughout the paper, we shall investigate regimes verifying

$$
\frac{\mathrm{VT}}{\mathrm{L}}=1
$$

Adopting the convention that dimensionless parameters will be denoted by Greek letters, we set

$$
\begin{cases}\eta=\frac{\mathcal{U}_{f l}}{\mathrm{~V}}=\frac{\mathcal{L}_{\text {cor }} \mathrm{T}}{\mathcal{T}_{\text {cor }} \mathrm{L}} & \text { (size of velocity fluctuation) } \\ \tau=\frac{\mathcal{T}_{\text {rel }}}{\mathrm{T}} & \text { (relaxation time) } \\ \varepsilon=\frac{\mathcal{T}_{\text {cor }}}{\mathrm{T}} \text { and } \quad \lambda=\frac{\mathcal{L}_{f l}}{\mathrm{~L}} & \text { (time and space scales of turbulence). }\end{cases}
$$


We point out that contrary to the physical constants the choice of units is free. (It depends on the scale of observations.) Therefore, in the determination of the different regimes we will distinguish the physical assumptions which compare the physical constants and the assumptions related to a choice of units.

We associate to these quantities the following dimensionless variables

$$
\bar{t}=\frac{t}{\mathrm{~T}}, \quad \bar{x}=\frac{x}{\mathrm{~L}}, \quad \bar{v}=\frac{v}{\mathrm{~V}}=\frac{v \mathrm{~T}}{\mathrm{~L}} .
$$

Finally, let us set

$$
\left\{\begin{array}{l}
\bar{f}(\bar{t}, \bar{x}, \bar{v})=f(\mathrm{~T} \bar{t}, \mathrm{~L} \bar{x}, \mathrm{~V} \bar{v}) \mathrm{L}^{N} \mathrm{~V}^{N}, \\
\bar{u}(\bar{t}, \bar{x})=\frac{1}{\mathcal{U}_{f l}} u_{f l}(\mathrm{~T} \bar{t}, \mathrm{~L} \bar{x})=U\left(\mathrm{~T} \bar{t} / \mathcal{T}_{c o r}, \mathrm{~L} \bar{x} / \mathcal{L}_{f l}\right) .
\end{array}\right.
$$

Hence, dropping the overlines, we are led to

$$
\partial_{t} f+v \cdot \nabla_{x} f+\frac{1}{\tau} \nabla_{v} \cdot((\eta u-v) f)=0
$$

with $u(t, x)=U(t / \varepsilon, x / \lambda)$.

\subsection{Asymptotic regimes}

In this work, we study the effects of turbulence, characterized by the limit $\varepsilon \rightarrow 0$, in two different physical situations. The former consists of assuming $\tau \ll 1$ which corresponds to a "Fine Particles Regime". Indeed in this regime, the Stokes force which is a friction term is predominant. It presupposes that the particles are "light" (or "fine") compared to the surrounding fluid. It results that the kinetic velocities relax rapidly to the velocity of the fluid. We are thus led to fluid equations for the (macroscopic) concentration of particles. Therefore this situation corresponds to a "Fluid Regime".

The latter assumes $\tau=1$ which corresponds to a "High Inertia Particles Regime" since the particles are less influenced by the turbulence and they still follow their individual trajectories. The effective equation remains kinetic and the turbulence leads to a diffusion process of the velocities of the particles.

\subsubsection{Fine particles regimes}

Let us first assume that $\tau \ll 1$. This scaling is reminiscent to hydrodynamic limits in rarefied gas dynamics, see e.g. [10]. When dealing with such a regime, we might expect a limit equation concerning averaged macroscopic quantities. It is then convenient to use the moment equations associated to (2). We set

$$
\rho(t, x)=\int_{\mathbb{R}^{N}} f \mathrm{~d} v, \quad J(t, x)=\int_{\mathbb{R}^{N}} v f \mathrm{~d} v, \quad \mathbb{P}(t, x)=\int_{\mathbb{R}^{N}} v \otimes v f \mathrm{~d} v .
$$

Integration of (2) with respect to the velocity variable yields the following system

$$
\begin{gathered}
\partial_{t} \rho(t, x)+\operatorname{div}_{x} J=0, \\
\partial_{t} J+\operatorname{div}_{x} \mathbb{P}=\frac{1}{\tau}(\eta \rho u-J) .
\end{gathered}
$$

In such a regime, we can formally guess that the solution looks like a Dirac mass centred to velocity $v=\eta u$, with density $\rho(t, x)$. We expect that

$$
f(t, x, v) \sim \rho(t, x) \delta_{v=\eta u(t, x)}
$$

the macroscopic concentration $\rho$ being of order 1. Accordingly, we have

$$
\rho=\mathcal{O}(1), \quad J=\mathcal{O}(\eta), \quad \mathbb{P}=\mathcal{O}\left(\eta^{2}\right)
$$


Furthermore, (6) implies that $(\rho \eta u-J) \rightarrow 0$, so that multiplying (5) by $\tau$ we deduce $\tau J \rightarrow 0$ and $\tau \mathbb{P} \rightarrow 0$. We are thus led to the conditions

$$
\tau \eta, \quad \tau \eta^{2} \rightarrow 0
$$

We also have to average over turbulence which imposes $\varepsilon \rightarrow 0$. Now, we can distinguish two approaches, which lead to investigate different asymptotic regimes.

\section{- Small Stokes number regime}

We shall see within the proofs that turbulence induces an effective drift term of order $\mathcal{O}\left(\varepsilon \eta^{2} / \lambda\right)$ and an effective diffusion coefficient of order $\mathcal{O}\left(\varepsilon \eta^{2}\right)$. The regimes where both effects account are thus characterized by

$$
\varepsilon \rightarrow 0, \quad \varepsilon \eta^{2}=\lambda, \quad \lambda=\mathcal{O}(1)
$$

Remark that it implies $\eta \rightarrow \infty$.

Coming back to the physical constants, these conditions mean that the observation time scale $\mathrm{T}$ is large compared to both the relaxation time $\mathcal{T}_{\text {rel }}$ and the time scale of turbulence $\mathcal{T}_{\text {cor }}$. Furthermore, in this regime the Stokes number defined by the ratio

$$
\frac{\text { relaxation time }}{\text { time scale of turbulence }}=\frac{\tau}{\varepsilon}=\mathcal{O}\left(\tau \eta^{2}\right) \rightarrow 0
$$

is small: we have $\mathrm{T} \gg \mathcal{T}_{\text {cor }} \gg \mathcal{T}_{\text {rel }}$. On the other hand, we note that the conditions imply $\mathrm{L} \approx \mathcal{L}_{f l} \gg \mathcal{L}_{\text {cor }}$ while time and length units are related by

$$
\mathrm{L}=\frac{\mathcal{L}_{c o r}^{2}}{\mathcal{L}_{f l}} \frac{\mathrm{T}}{\mathcal{T}_{\text {cor }}} .
$$

The same analysis also applies to the situation where convection dominates diffusion in the asymptotic regime. It corresponds to the case

$$
\lambda \rightarrow 0, \quad \mathrm{~L} \gg \mathcal{L}_{f l} \gg \mathcal{L}_{\text {cor }}
$$

Summarizing the discussion, we wish to investigate asymptotic regimes verifying

$$
\frac{\varepsilon \eta^{2}}{\lambda}=1, \quad \lambda \gg \varepsilon \gg \tau, \quad \lambda \rightarrow \lambda_{\infty} \in[0, \infty)
$$

The corresponding result is the object of Theorem 1.

- Large Stokes number regime

We shall see that another fluid regime, i.e. where $\tau \rightarrow 0$, can be dealt with, assuming that the Stokes number is large:

$$
\frac{\tau}{\varepsilon} \rightarrow \infty
$$

The leading term will be in this situation a convective term, whose order is $\mathcal{O}\left(\frac{\varepsilon^{2} \eta^{2}}{\tau \lambda}\right)$. Hence, we naturally assume

$$
\varepsilon^{2} \eta^{2}=\tau \lambda
$$

which will be combined with the assumptions $\varepsilon / \lambda \rightarrow 0$ and, as mentioned above, $\tau \eta^{2} \rightarrow 0$. Summarizing, we wish to investigate the asymptotic regime where the dimensionless coefficients satisfy

$$
\frac{\tau}{\varepsilon} \rightarrow \infty, \quad \varepsilon^{2} \eta^{2}=\tau \lambda, \quad \frac{\varepsilon}{\lambda} \rightarrow 0, \quad \tau \eta^{2} \rightarrow 0 .
$$


Note that these conditions have the following consequences:

$-\eta^{2}=\frac{\lambda}{\varepsilon} \frac{\tau}{\varepsilon} \rightarrow \infty$

- hence, since $\tau \eta^{2}=\frac{\tau^{2}}{\varepsilon^{2}} \lambda$ tends to 0 , it follows that $\tau \rightarrow 0$ and $\lambda \rightarrow 0$.

Coming back to the physical variables, these assumptions mean that

$$
\mathrm{T} \gg \mathcal{T}_{\text {rel }} \gg \mathcal{T}_{\text {cor }}
$$

combined to

$$
\mathrm{L} \gg \mathcal{L}_{f l} \gg \mathcal{L}_{\text {cor }}
$$

The corresponding result is the object of Theorem 2.

Remark 1. Note that any asymptotic regime with $\eta \rightarrow 0$ would lead to the trivial limit equation $\partial_{t} \rho=0$.

\subsubsection{High-inertia-particles regime}

In this regime, the velocity of the particles can differ from the velocity of the surrounding fluid. It means that the particles have enough inertia to balance the Stokes force. Actually, the relaxation time scale remains large compared to the characteristics time scale of correlations. On the other hand, the fluctuations have large amplitude. In such a physical situation, we keep a kinetic description, obtaining when passing to the limit a Vlasov-Fokker-Planck equation which involves a diffusion matrix defined from the properties of the turbulent fluctuations. Precisely, we assume

$$
\tau=\mathcal{O}(1), \quad \varepsilon \rightarrow 0 .
$$

We shall see that turbulence induces now an effective diffusion coefficient of order $\mathcal{O}\left(\varepsilon \eta^{2}\right)$ and an effective drift of order $\mathcal{O}\left(\varepsilon^{2} \eta^{2} / \lambda\right)$. This motivates to suppose

$$
\varepsilon \eta^{2}=\mathcal{O}(1), \quad \frac{\varepsilon^{2} \eta^{2}}{\lambda}=\frac{\varepsilon}{\lambda} \varepsilon \eta^{2}=\mathcal{O}(1)
$$

which implies

$$
\eta=\mathcal{O}\left(\frac{1}{\sqrt{\varepsilon}}\right) \rightarrow \infty, \quad \lambda=\mathcal{O}(\varepsilon) \rightarrow 0 .
$$

Coming back to the physical quantities, this scaling means that the observation time scale and the relaxation time have the same order but they are large compared to the time scale of turbulence

$$
\mathrm{T} \approx \mathcal{T}_{\text {rel }} \gg \mathcal{T}_{\text {cor }}
$$

In turn, the Stokes number $\tau / \varepsilon=\mathcal{T}_{\text {rel }} / \mathcal{T}_{\text {cor }}$ is large. On the other hand, both length scales of turbulence are small compared to the length unit, with the scaling

$$
\mathrm{L} \gg \mathcal{L}_{c o r} \gg \mathcal{L}_{f l} .
$$

The corresponding statement is given in Theorem 3.

\section{MAin Results}

This section is devoted to the statement of the main results of the paper. Let us start by recalling some classical notations and basic facts from probability theory.

Let $\Omega$ stands for an abstract space. It is endowed with a $\sigma$-algebra $\mathcal{P}$ and a probability measure $\mathbb{P}$. A random variable is a measurable function $X: \Omega \rightarrow \mathbb{R}$. Actually, we shall consider families of random variables $X(z)$ parameterized by a variable $z$ which belongs to a certain set $Z$. Such a function will be said "deterministic" 
when it does not depend on the variable $\omega \in \Omega$. Next, let $X$ be an integrable random variable. Its expectation is defined by

$$
\mathbb{E}(X)=\int_{\Omega} X(\omega) \mathrm{d} \mathbb{P}(\omega)
$$

In what follows, as usual when dealing with stochastic processes, we will never write the dependence with respect to the random parameter $\omega$.

Next, we need to describe the set of assumptions fulfilled by the data.

\subsection{Assumptions on the data}

The velocity field $u$ presents random oscillations with respect to time and space variables; we suppose it has the form

where the function $U$ is required to satisfy

$$
u(t, x)=U(t / \varepsilon, x / \lambda) \in \mathbb{R}^{N},
$$

$$
\left\{\begin{array}{l}
\text { The random velocity field } U \in \mathrm{L}^{\infty}\left(\mathbb{R} ; \mathrm{W}^{2, \infty}\left(\mathbb{R}^{N}\right)\right) \text { satisfies for some } K>0 \\
\sup _{t, x}\left|\partial_{x}^{\alpha} U(t, x)\right| \leq K<\infty, \text { almost surely for any } \alpha \in \mathbb{N}^{N},|\alpha| \leq 2
\end{array}\right.
$$

It implies that $U(t, x)$ is a bounded random variable. We assume

$$
\left\{\begin{array}{l}
\bullet \mathbb{E}(U(t, x))=0 ; \\
\bullet \text { there exists a }\left(N \times N \text { matrix valued) function } R:[0, \infty) \times \mathbb{R}^{N} \rightarrow \mathcal{M}_{N},\right. \\
\text { such that for every } x, y \in \mathbb{R}^{N}, \text { for every } t, s \in[0, \infty): \\
\mathbb{E}(U(t, x) \otimes U(s, y))=R(t-s, x-y) ; \\
\bullet \text { for every } x, y \in \mathbb{R}^{N}, \text { for every } t, s \in[0, \infty), \text { if }|t-s| \geq 1, \\
\text { then } U(t, x) \text { and } U(s, y) \text { are independent random variables. }
\end{array}\right.
$$

Here, $\mathcal{M}_{N}$ stands for the space of $N \times N$ matrices, while for vectors $a$ and $b$ in $\mathbb{R}^{N}, a \otimes b$ stands for the matrix having coefficients $a_{i} b_{j}$. Various examples of velocity fields which fulfil this set of assumptions are described in [23], or [2]. The first assumption means that $U$ is centred. The second assumption means that $U$ is stationary: the covariances of $U$ evaluated at different points depends only on the differences between these points. The third assumption can be seen as a Markov-like assumption on the trajectories.

Remark 2. With assumption (9), events are decorrelated when occurring at times separated by $\varepsilon \rightarrow 0$, which is reminiscent to the classical Kubo's analysis, [20], see also [19].

Remark 3. The derivation of the model and in particular the use of the Stokes force require the incompressibility of the surrounding fluid. However, we can consider perturbations of the Stokes force by other forces (e.g. electrostatic forces, magnetic forces...). Such perturbations can be incorporated in the field $u$ which can be no more incompressible. For this reason, we consider in this work general fields $u$.

Since $u$ is a random function, the solution $f$ of (2) is also a random function. However, we shall assume that the initial data remains deterministic. Precisely, we suppose

$$
f_{0}: \mathbb{R}^{N} \times \mathbb{R}^{N} \rightarrow \mathbb{R} \text { is deterministic, } \quad f_{0} \geq 0 .
$$

Then, the cornerstone of our analysis relies on the following independency statement.

Lemma 1. We suppose the initial data $f_{0}$ satisfies (10). Let $f$ be the solution of (2) with the Cauchy data $f_{\mid t=0}=f_{0}$. Then for every $t \geq 0, f(t)$ is non negative, and, furthermore, $f(t)$ and $u(s)$ are independent provided $s \geq t+\varepsilon$. 
It is an immediate consequence of the fact that $f_{0}$ is deterministic. Then for any $t \geq 0, f(t)$ depends only on the realization of $u(s)$ for $s \in[0, t]$. The third assumption of (9) leads to the result.

\subsection{The fine particles regime}

Now, we state the results which correspond to the physical situation described in Section 2.2.1.

Theorem 1. Assume (8), (9). Suppose

$$
\varepsilon \eta^{2}=\lambda, \quad \lambda \gg \varepsilon \gg \tau, \quad \lambda \rightarrow \lambda_{\infty} \in[0, \infty)
$$

Let the initial data $f_{0}$ satisfy (10) and

$$
\int_{\mathbb{R}^{N}} \int_{\mathbb{R}^{N}} f_{0} \mathrm{~d} v \mathrm{~d} x=M_{0}<\infty, \quad \int_{\mathbb{R}^{N}} \int_{\mathbb{R}^{N}} \frac{v^{2}}{2} f_{0} \mathrm{~d} v \mathrm{~d} x=M_{2}<\infty .
$$

Let $f$ be the solution of (2) with the Cauchy data $f_{\mid t=0}=f_{0}$. Set $\rho(t, x)=\int f(t, x, v) \mathrm{d} v$. Let $0<T<\infty$. Then, up to a subsequence, $\mathbb{E}(\rho)$ converges in $\mathrm{C}^{0}\left([0, T] ; \mathcal{M}^{1}\left(\mathbb{R}^{N}\right)-\right.$ weak $\left.-\star\right)$ to $r$ solution of the following drift-diffusion equation

$$
\left\{\begin{array}{l}
\partial_{t} r+\operatorname{div}\left(c r-\lambda_{\infty} D \nabla_{x} r\right)=0 \\
r_{\mid t=0}=\int_{\mathbb{R}^{N}} f_{0} \mathrm{~d} v
\end{array}\right.
$$

The diffusivity matrix $D$ and the drift vector $c$ are defined by

$$
\left\{\begin{array}{l}
D_{i j}=\int_{0}^{1} R_{i j}(z, 0) \mathrm{d} z \\
c_{i}=\int_{0}^{1} \sum_{j=1}^{N}\left(\partial_{y_{j}} R_{i j}\right)(z, 0) \mathrm{d} z .
\end{array}\right.
$$

The matrix $D$ satisfies, for any $\xi \in \mathbb{R}^{N} \backslash\{0\}$

$$
\sum_{i, j=1}^{N} D_{i j} \xi_{i} \xi_{j} \geq 0
$$

Remark 4. The limit $r$ solves the drift-diffusion equation in the sense that

$$
\frac{\mathrm{d}}{\mathrm{d} t} \int_{\mathbb{R}^{N}} \psi r \mathrm{~d} x=\int_{\mathbb{R}^{N}}\left(\operatorname{div}\left(\lambda_{\infty} D^{T} \nabla_{x} \psi\right)+c \cdot \nabla_{x} \psi\right) r \mathrm{~d} x
$$

holds for any $\psi \in \mathrm{C}_{c}^{\infty}\left(\mathbb{R}^{N}\right)$, these quantities belonging to $\mathrm{C}^{0}([0, T])$. Remark the limit equation is purely convective when $\lambda \rightarrow 0$.

Remark 5. Assumption (9) can be slightly modified so that we obtain space dependent effective coefficients. For instance consider random velocity fields verifying $\mathbb{E}(U(t, x) \otimes U(s, y))=R(t-s, x, y)$ with $R: \mathbb{R} \times \mathbb{R}^{N} \times \mathbb{R}^{N} \rightarrow$ $\mathcal{M}_{N}$ a smooth function, compactly supported in $(-1,+1) \times \mathbb{R}^{N} \times \mathbb{R}^{N}$.

Theorem 2. Assume (8), (9). Suppose

$$
\varepsilon^{2} \eta^{2}=\tau \lambda, \quad \lambda \gg \varepsilon, \quad \tau \gg \varepsilon, \quad \tau \eta^{2} \rightarrow 0
$$


Let the initial data $f_{0}$ satisfy (10) and (12). Let $f$ be the solution of (2) with the Cauchy data $f_{\mid t=0}=f_{0}$. Set $\rho(t, x)=\int f(t, x, v) \mathrm{d} v$. Let $0<T<\infty$. Then, up to a subsequence, $\mathbb{E}(\rho)$ converges in $\mathrm{C}^{0}\left([0, T] ; \mathcal{M}^{1}\left(\mathbb{R}^{N}\right)-\right.$ weak $-\star)$ to $r$ solution of the following convection equation

$$
\left\{\begin{array}{l}
\partial_{t} r+\operatorname{div}(c r)=0 \\
r_{\mid t=0}=\int_{\mathbb{R}^{N}} f_{0} \mathrm{~d} v
\end{array}\right.
$$

with

$$
c_{i}=\int_{0}^{1} \sum_{j=1}^{N}\left(\partial_{y_{j}} R_{i j}\right)(z, 0) z \mathrm{~d} z .
$$

\subsection{The high inertia particles regime}

Here, we give the statement corresponding to the physical framework introduced in Section 2.2.2.

Theorem 3. Assume (8), (9). Suppose

$$
\tau=1, \quad \varepsilon \rightarrow 0, \quad \eta=1 / \sqrt{\varepsilon}, \quad \frac{\varepsilon}{\lambda} \rightarrow \kappa_{\infty} \in[0, \infty) .
$$

Let the initial data $f_{0} \geq 0$ satisfy (10) and (12). Let $f$ be the solution of (2) with the Cauchy data $f_{\mid t=0}=f_{0}$. Let $0<T<\infty$. Then, up to a subsequence, $\mathbb{E}(f)$ converges in $\mathrm{C}^{0}\left([0, T], \mathcal{M}^{1}\left(\mathbb{R}^{N} \times \mathbb{R}^{N}\right)-\right.$ weak $\left.-\star\right)$ to $F$ solution, in $\mathcal{D}^{\prime}\left([0, \infty) \times \mathbb{R}^{N}\right)$ of the following Fokker-Planck equation

$$
\left\{\begin{array}{l}
\partial_{t} F+v \cdot \nabla_{x} F-\nabla_{v} \cdot\left(D(v) \nabla_{v} F+v F\right)=0, \\
F_{\mid t=0}=f_{0} .
\end{array}\right.
$$

The matrix $D$ is defined by

$$
D_{i j}(v)=\int_{0}^{1} R_{i j}\left(z, \kappa_{\infty} z v\right) \mathrm{d} z
$$

For any $\xi \in \mathbb{R}^{N}$, it satisfies

$$
\sum_{i j=1}^{N} D_{i j} \xi_{i} \xi_{j} \geq 0
$$

This result is quite close to the analysis performed by Clouet-Domelevo [5]. There, the random fluctuations are defined through a piecewise constant velocity field. In each time step, the field is an identically distributed random variable which follows a Gaussian law. Then the so-called white noise limit is investigated where the size $\varepsilon$ of the time intervals shrinks.

\section{Proofs}

This section is devoted to the proofs of Theorems 1, 2 and 3. As usual, we start with some basic estimates in suitable functional spaces. Then, we explain how we can pass to the limit in the equation.

\subsection{Key estimates}

Solution of (2) satisfy some useful estimates. First of all, the total mass is clearly conserved

$$
\int_{\mathbb{R}^{N}} \int_{\mathbb{R}^{N}} f(t, x, v) \mathrm{d} v \mathrm{~d} x=\int_{\mathbb{R}^{N}} \int_{\mathbb{R}^{N}} f_{0}(x, v) \mathrm{d} v \mathrm{~d} x=M_{0} .
$$


In view of (12) this quantity is bounded uniformly with respect to the parameters $\tau, \varepsilon, \eta, \lambda$, so that one has at least a uniform estimate on the solution in $\mathrm{L}^{\infty}\left(\mathbb{R}^{+} ; \mathrm{L}^{1}\left(\mathbb{R}^{N} \times \mathbb{R}^{N}\right)\right)$. Next, (2) can be rewritten as

$$
\partial_{t} f+v \cdot \nabla_{x} f-\frac{N}{\tau} f+\frac{1}{\tau}(\eta u-v) \cdot \nabla_{v} f=0 .
$$

This non conservative form of the equation allows to obtain estimates in $\mathrm{L}^{\infty}\left(0, T ; \mathrm{L}^{p}\left(\mathbb{R}^{N} \times \mathbb{R}^{N}\right)\right)$. However, the estimates are not uniform with respect to the parameter $\tau$. Contrarily, as $\tau \rightarrow 0$, as explained before, one may expect that $f$ concentrates in velocity to the velocity of the fluid $f(t, x, v) \sim \rho(t, x) \delta(v=\eta u)$ (see for instance for such concentration effects $[16,17])$.

For further purposes, it is convenient to look at the evolution of the kinetic energy. We have

$$
\begin{aligned}
\frac{\mathrm{d}}{\mathrm{d} t} \int_{\mathbb{R}^{N}} \int_{\mathbb{R}^{N}} \frac{v^{2}}{2} f(t) \mathrm{d} v \mathrm{~d} x= & -\int_{\mathbb{R}^{N}} \int_{\mathbb{R}^{N}} \frac{v^{2}}{2} \frac{1}{\tau} \nabla_{v} \cdot((\eta u-v) f(t)) \mathrm{d} v \mathrm{~d} x \\
= & -\frac{1}{\tau} \int_{\mathbb{R}^{N}} \int_{\mathbb{R}^{N}} v^{2} f(t) \mathrm{d} v \mathrm{~d} x+\frac{\eta}{\tau} \int_{\mathbb{R}^{N}} \int_{\mathbb{R}^{N}} u \cdot v f(t) \mathrm{d} v \mathrm{~d} x \\
\leq & -\frac{1}{\tau} \int_{\mathbb{R}^{N}} \int_{\mathbb{R}^{N}} v^{2} f(t) \mathrm{d} v \mathrm{~d} x \\
& +\frac{\eta}{\tau}\|u\|_{L^{\infty}}\left(\int_{\mathbb{R}^{N}} \int_{\mathbb{R}^{N}} f(t) \mathrm{d} v \mathrm{~d} x\right)^{1 / 2}\left(\int_{\mathbb{R}^{N}} \int_{\mathbb{R}^{N}} v^{2} f(t) \mathrm{d} v \mathrm{~d} x\right)^{1 / 2} .
\end{aligned}
$$

Let us set $Y(t)=\left(\iint v^{2} f(t) \mathrm{d} v \mathrm{~d} x\right)^{1 / 2}$. It thus verifies the differential inequality

$$
\frac{\mathrm{d}}{\mathrm{d} t}\left(\mathrm{e}^{t / \tau} Y\right) \leq M_{0} \frac{\eta}{\tau}\|u\|_{L^{\infty}} \mathrm{e}^{t / \tau}
$$

We deduce that $Y(t) \leq \mathrm{e}^{-t / \tau} Y(0)+M_{0} \eta\|u\|_{L^{\infty}}$. We have proved the following statement.

Lemma 2. Suppose (8). Let the initial data $f_{0} \geq 0$ satisfy (10), (12). Then, for any timet $\geq 0, \iint f(t) \mathrm{d} v \mathrm{~d} x=$ $M_{0}$ and there exists a constant $C>0$, depending only on $M_{0}, M_{2}$ and $K$ such that, almost surely, for any $t \geq 0$,

$$
\int_{\mathbb{R}^{N}} \int_{\mathbb{R}^{N}}|v| f(t) \mathrm{d} v \mathrm{~d} x \leq C \eta, \quad \int_{\mathbb{R}^{N}} \int_{\mathbb{R}^{N}} v^{2} f(t) \mathrm{d} v \mathrm{~d} x \leq C \eta^{2} .
$$

The estimate on $|v| f$ follows from the mass and the energy estimates and an application of the Cauchy-Schwarz inequality. Lemma 2 provides readily estimates on the macroscopic moments defined in (3) which in turn justify (7).

Corollary 1. There exists a constant $C>0$, depending only on $M_{0}, M_{2}, K$ such that almost surely, for any $t \geq 0$

$$
\int_{\mathbb{R}^{N}} \rho(t) \mathrm{d} x=M_{0}, \quad \int_{\mathbb{R}^{N}}|J|(t) \mathrm{d} x \leq C \eta, \quad \int_{\mathbb{R}^{N}}|\mathbb{P}| \mathrm{d} x \leq C \eta^{2}
$$

\subsection{Proof of Theorem 1}

For the proof of Theorem 1, we do not use the kinetic equation; instead, we work on the moments system (4-5). As a first consequence of Corollary 1, we have the following equicontinuity estimate. 
Lemma 3. Let $\psi \in \mathrm{C}_{b}^{1}\left(\mathbb{R}^{N}\right)$ and $h>0$. Then, we have

$$
\begin{aligned}
\left|\int_{\mathbb{R}^{N}} \rho(t+h) \psi \mathrm{d} x-\int_{\mathbb{R}^{N}} \rho(t) \psi \mathrm{d} x\right| & =h\left|\int_{0}^{1} \int_{\mathbb{R}^{N}} J(t+h s) \cdot \nabla \psi \mathrm{d} x\right| \\
& \leq C h \eta\|\nabla \psi\|_{L^{\infty}\left(\mathbb{R}^{N}\right)}
\end{aligned}
$$

where the constant $C$ depends only on the constants appearing in (12).

Notice that this estimate does not provide any compactness property on the sequence $\rho$ since in the scaling assumptions $\eta$ tends to $\infty$. To obtain more complete information, we shall use the second moment equation. Precisely, we rewrite (5) as follows

$$
J=\eta \rho u-\tau \partial_{t} J-\tau \operatorname{div}_{x} \mathbb{P} .
$$

In the sequel, the notation $\mathcal{O}(\beta)$ stands for a time dependent function (random or not) which is bounded by $C \beta$ where the constant $C>0$ does not depend on the time variable $t$, the random variable nor on the parameters $\varepsilon, \tau, \eta$ and $\lambda$.

Lemma 4. Let $\psi \in \mathrm{C}_{c}^{\infty}\left(\mathbb{R}^{N}\right)$. Then, we have

$$
\frac{\mathrm{d}}{\mathrm{d} t}\left(\int_{\mathbb{R}^{N}} \rho(t) \psi \mathrm{d} x+\mathcal{O}(\tau \eta)\right)=\eta \int_{\mathbb{R}^{N}} \rho(t) u(t) \cdot \nabla \psi \mathrm{d} x+\mathcal{O}\left(\tau \eta^{2}\right) .
$$

Proof. Using (4) and (17) we obtain (using integration by parts)

$$
\begin{aligned}
\frac{\mathrm{d}}{\mathrm{d} t}\left(\int_{\mathbb{R}^{N}} \rho(t) \psi \mathrm{d} x\right)= & \int_{\mathbb{R}^{N}} J(t) \cdot \nabla \psi \mathrm{d} x \\
= & \eta \int_{\mathbb{R}^{N}} \rho(t) u(t) \cdot \nabla \psi \mathrm{d} x \\
& -\tau \frac{\mathrm{d}}{\mathrm{d} t}\left(\int_{\mathbb{R}^{N}} J(t) \cdot \nabla \psi \mathrm{d} x\right)+\tau \int_{\mathbb{R}^{N}} \mathbb{P}(t): D_{x}^{2} \psi \mathrm{d} x
\end{aligned}
$$

The estimates of Corollary 1 conclude the proof.

We recall that, for the asymptotic regime we are dealing with, $\tau \eta$ and $\tau \eta^{2}$ tend to 0 . Hence, we are left with the task of determining the limit of $\eta \int \rho(t) u(t) \cdot \nabla \psi \mathrm{d} x$. Actually, this term is not bounded (recall that $\eta \rightarrow \infty)$; but its expectation is bounded. The strategy to compute the limit of the expectation is inspired from [23]. We express $\rho(t)$ with $\rho(t-\varepsilon)$ in order to obtain a decorellation with $u(t)$. Namely, we write, using (4)

$$
\rho(t)=\rho(t-\varepsilon)-\varepsilon \int_{0}^{1} \operatorname{div}_{x} J(t-\varepsilon s) \mathrm{d} s .
$$

We are thus able to prove the following statement (which justifies the discussion on the scaling of the parameters).

Lemma 5. Let $\psi \in \mathrm{C}_{c}^{\infty}\left(\mathbb{R}^{N}\right)$. Then, for any $t \geq \varepsilon$, we have

$$
\begin{aligned}
\eta \mathbb{E} \int_{\mathbb{R}^{N}} \rho(t) u(t) \cdot \nabla \psi \mathrm{d} x & =\eta \varepsilon \mathbb{E} \int_{\mathbb{R}^{N}} \int_{0}^{1} J(t-\varepsilon s) \cdot \nabla(u(t) \cdot \nabla \psi) \mathrm{d} s \mathrm{~d} x \\
& =\mathcal{O}\left(\frac{\varepsilon \eta^{2}}{\lambda}\right)+\mathcal{O}\left(\varepsilon \eta^{2}\right) .
\end{aligned}
$$

As a consequence, we establish the following compactness property. 
Corollary 2. Let $\varepsilon_{n}, \tau_{n}, \lambda_{n}, \eta_{n}$ be sequences of parameters satisfying (11). Let $f_{n}$ be the associated solution of (2) with concentration $\rho_{n}(t, x)=\int f_{n} \mathrm{~d} v$. Let $\psi \in \mathrm{C}_{c}^{\infty}\left(\mathbb{R}^{N}\right)$. Then, for every $T>0$, the family of functions

$$
\left\{\mathbb{E} \int_{\mathbb{R}^{N}} \rho_{n}(t) \psi \mathrm{d} x, n \in \mathbb{N}\right\}
$$

is relatively compact in $\mathrm{C}^{0}([0, T])$.

Proof of Lemma 5. Multiply (19) by $\eta u(t) \cdot \nabla \psi$ and integrate. We get

$$
\eta \int_{\mathbb{R}^{N}} \rho(t) u(t) \cdot \nabla \psi \mathrm{d} x=\eta \int_{\mathbb{R}^{N}} \rho(t-\varepsilon) u(t) \cdot \nabla \psi \mathrm{d} x+\eta \varepsilon \int_{\mathbb{R}^{N}} \int_{0}^{1} J(t-\varepsilon s) \cdot \nabla(u(t) \cdot \nabla \psi) \mathrm{d} s \mathrm{~d} x .
$$

Combining (9) and Lemma 1 we realize that the expectation of the first term vanishes

$$
\eta \mathbb{E} \int_{\mathbb{R}^{N}} \rho(t-\varepsilon) u(t) \cdot \nabla \psi \mathrm{d} x=\eta \int_{\mathbb{R}^{N}} \mathbb{E}(\rho(t-\varepsilon)) \mathbb{E}(u(t)) \cdot \nabla \psi \mathrm{d} x=0 .
$$

Next, using (8) and Corollary 1, we obtain

$$
\eta \varepsilon \mathbb{E} \int_{\mathbb{R}^{N}} \int_{0}^{1} J(t-\varepsilon s) \cdot \nabla(u(t) \cdot \nabla \psi) \mathrm{d} s \mathrm{~d} x=\mathcal{O}\left(\eta \varepsilon \times \frac{\eta}{\lambda}\right)+\mathcal{O}(\eta \varepsilon \times \eta) .
$$

Assuming (11), we have $\frac{\eta^{2} \varepsilon}{\lambda}=\mathcal{O}(1)$, where $\lambda$ has a finite limit, so that $\eta^{2} \varepsilon=\mathcal{O}(1)$.

Proof of Corollary 2. Take the expectation of equation (18). Then, using Lemma 5, an application of the Arzela-Ascoli theorem shows that the sequence $\left\{\mathbb{E} \int \rho_{n}\left(t+\varepsilon_{n}\right) \psi \mathrm{d} x+\mathcal{O}\left(\tau_{n} \eta_{n}\right), n \in \mathbb{N}\right\}$ is relatively compact in $\mathrm{C}^{0}([0, T])$. (We have to shift the time because (20) holds only for $t \geq \varepsilon_{n}$.) Since $\tau_{n} \eta_{n} \rightarrow 0$, it follows that $\left\{\mathbb{E} \int \rho_{n}\left(t+\varepsilon_{n}\right) \psi \mathrm{d} x, n \in \mathbb{N}\right\}$ is also relatively compact in $\mathrm{C}^{0}([0, T])$. Then, we go back to Lemma 3 which yields

$$
\left|\int_{\mathbb{R}^{N}} \rho_{n}\left(t+\varepsilon_{n}\right) \psi \mathrm{d} x-\int_{\mathbb{R}^{N}} \rho_{n}(t) \psi \mathrm{d} x\right| \leq C \varepsilon_{n} \eta_{n}\|\nabla \psi\|_{L^{\infty}\left(\mathbb{R}^{N}\right)} .
$$

The fact that $\varepsilon_{n} \eta_{n} \rightarrow 0$ ends the proof.

Having obtained the compactness of $\mathbb{E} \rho$, in a suitable weak sense, it remains to pass to the limit in the equation. By using (18) and (20), we get

$$
\frac{\mathrm{d}}{\mathrm{d} t}\left(\mathbb{E} \int_{\mathbb{R}^{N}} \rho(t) \psi \mathrm{d} x+\mathcal{O}(\tau \eta)\right)=\eta \varepsilon \mathbb{E} \int_{\mathbb{R}^{N}} \int_{0}^{1} J(t-\varepsilon s) \cdot \nabla(u(t) \cdot \nabla \psi) \mathrm{d} s \mathrm{~d} x+\mathcal{O}\left(\tau \eta^{2}\right) .
$$

Then, we use (17) again. Consider a function $\phi \in\left[\mathrm{C}_{b}^{1}\left([0, \infty) \times \mathbb{R}^{N}\right)\right]^{N}$, possibly depending on the parameters and on the random variable. We have

$$
\begin{aligned}
\int_{0}^{1} \int_{\mathbb{R}^{N}} J(t-\varepsilon s) \cdot \phi(t) \mathrm{d} x \mathrm{~d} s= & \eta \int_{0}^{1} \int_{\mathbb{R}^{N}} \rho(t-\varepsilon s) u(t-\varepsilon s) \cdot \phi(t) \mathrm{d} x \mathrm{~d} s \\
& -\tau \frac{\mathrm{d}}{\mathrm{d} t} \int_{0}^{1} \int_{\mathbb{R}^{N}} J(t-\varepsilon) \cdot \phi(t) \mathrm{d} x \mathrm{~d} s \\
& +\tau \int_{0}^{1} \int_{\mathbb{R}^{N}} J(t-\varepsilon s) \cdot \partial_{t} \phi(t) \mathrm{d} x \mathrm{~d} s \\
& +\tau \int_{0}^{1} \int_{\mathbb{R}^{N}} \mathbb{P}(t-\varepsilon s): \nabla \phi(t) \mathrm{d} x \mathrm{~d} s .
\end{aligned}
$$


The three last integrals can be bounded by

$$
C \tau \eta\left\|\partial_{t} \phi\right\|_{\infty}, \quad C \tau \eta^{2}\|\nabla \phi\|_{\infty}, \quad C \tau \eta\|\phi\|_{\infty}
$$

respectively, the constant $C$ depending only on $M_{0}, M_{2}$ of (12). Inserting this expression with $\phi=\eta \varepsilon \nabla(u(t)$. $\nabla \psi)$ in $(21)$ we are led to the following lemma.

Lemma 6. Let $\psi \in \mathrm{C}_{c}^{\infty}\left(\mathbb{R}^{N}\right)$ then

$$
\begin{aligned}
\frac{\mathrm{d}}{\mathrm{d} t}\left(\mathbb{E} \int_{\mathbb{R}^{N}} \rho(t) \psi \mathrm{d} x+\mathcal{O}\left(\tau \eta+\tau \eta^{2} \varepsilon / \lambda\right)\right)= & \varepsilon \eta^{2} \mathbb{E} \int_{0}^{1} \int_{\mathbb{R}^{N}} \rho(t-\varepsilon s) u(t-\varepsilon s) \cdot \nabla(u(t) \cdot \nabla \psi) \mathrm{d} s \mathrm{~d} x \\
& +\mathcal{O}\left(\tau \eta^{2}+\tau \eta^{2} / \lambda+\varepsilon \tau \eta^{3} / \lambda^{2}\right)
\end{aligned}
$$

Proof. The conclusion follows from the previous manipulations and remarking that, with $\phi=\eta \varepsilon \nabla(u(t) \cdot \nabla \psi)$, the following estimates hold

$$
\|\phi\|_{\infty} \leq C \varepsilon \eta / \lambda, \quad\left\|\partial_{t} \phi\right\|_{\infty} \leq C \eta / \lambda, \quad\|\nabla \phi\|_{\infty} \leq C \varepsilon \eta / \lambda^{2}
$$

Assuming (11), we observe that all the quantities

$$
\tau \eta, \quad \tau \eta^{2}, \quad \varepsilon \frac{\tau \eta^{2}}{\lambda}, \quad \frac{\tau \eta^{2}}{\lambda}, \quad \frac{\tau \varepsilon \eta^{3}}{\lambda^{2}}
$$

tend to 0 . Hence, we are left to the task of studying the leading term

$$
\mathbb{E} \int_{0}^{1} \int_{\mathbb{R}^{N}} \rho(t-\varepsilon s) \Phi(t, s, x) \mathrm{d} s \mathrm{~d} x
$$

with the notation

$$
\Phi(t, s, x)=\varepsilon \eta^{2} u(t-\varepsilon s, x) \cdot \nabla(u(t, x) \cdot \nabla \psi(x)) .
$$

It can be rewritten as follows

$$
\begin{aligned}
\mathbb{E} \int_{\mathbb{R}^{N}} \int_{0}^{1} \rho(t-\varepsilon s) \Phi(t, s) \mathrm{d} s \mathrm{~d} x= & \mathbb{E} \int_{\mathbb{R}^{N}} \int_{0}^{1} \mathbb{E}(\rho(t)) \Phi(t, s) \mathrm{d} s \mathrm{~d} x \\
& +\mathbb{E} \int_{\mathbb{R}^{N}} \int_{0}^{1}(\mathbb{E}(\rho(t-2 \varepsilon)-\rho(t))+\rho(t-\varepsilon s)-\rho(t-2 \varepsilon)) \Phi(t, s) \mathrm{d} s \mathrm{~d} x \\
& +\mathbb{E} \int_{\mathbb{R}^{N}} \int_{0}^{1}(\rho(t-2 \varepsilon)-\mathbb{E}(\rho(t-2 \varepsilon))) \Phi(t, s) \mathrm{d} s \mathrm{~d} x
\end{aligned}
$$

Actually, the last integral of the right hand side vanishes by using the independency property Lemma 1 which implies that $\mathbb{E}(\rho(t-2 \varepsilon) \Phi(t, s))=\mathbb{E} \rho(t-2 \varepsilon) \mathbb{E} \Phi(t, s)$. Next, the second integral can be evaluated by using Lemma 3; it is shown to be $\mathcal{O}\left(\varepsilon \eta^{2} \times \varepsilon \eta \times \lambda^{-2}\right)=\mathcal{O}\left(\left(\varepsilon \eta^{2} / \lambda\right)^{2} \times \eta^{-1}\right)=\mathcal{O}(1 / \eta) \rightarrow 0$. Therefore, it remains to pass to the limit in

$$
\int_{\mathbb{R}^{N}} \int_{0}^{1} \mathbb{E}(\rho(t)) \mathbb{E} \Phi(t, s) \mathrm{d} s \mathrm{~d} x
$$


where, by using assumption (9), we note that

$$
\begin{aligned}
\mathbb{E} \Phi(t, s) & =\varepsilon \eta^{2} \sum_{i, j} \mathbb{E}\left(u_{i}(t-\varepsilon s) \partial_{x_{i}}\left(u_{j}(t) \partial_{x_{j}} \psi\right)\right) \\
& =\varepsilon \eta^{2} \sum_{i, j}\left\{\partial_{x_{i}}\left(\mathbb{E}\left(u_{i}(t-\varepsilon s) u_{j}(t)\right) \partial_{x_{j}} \psi\right)-\mathbb{E}\left(\partial_{x_{i}} u_{i}(t-\varepsilon s) u_{j}(t)\right) \partial_{x_{j}} \psi\right\} \\
& =\varepsilon \eta^{2} \sum_{i, j} \partial_{x_{i}}\left(R_{j, i}(s, 0) \partial_{x_{j}} \psi\right)-\frac{\varepsilon \eta^{2}}{\lambda} \sum_{i, j} \partial_{x_{i}} R_{j, i}(s, 0) \partial_{x_{j}} \psi
\end{aligned}
$$

Since we assume $\varepsilon \eta^{2} / \lambda=1$, we get

$$
\mathbb{E} \int_{\mathbb{R}^{N}} \int_{0}^{1} \rho(t-\varepsilon s) \phi(t, s) \mathrm{d} s \mathrm{~d} x=\int_{\mathbb{R}^{N}} \mathbb{E}(\rho(t))\left(\operatorname{div}\left(\lambda D^{T} \nabla \psi\right)-c \cdot \nabla \psi\right)+\mathcal{O}(1 / \eta),
$$

with the matrix $D$ and the vector $c$ defined in Theorem 1 .

This equation combined together with Lemma 6 yields

$\frac{\mathrm{d}}{\mathrm{d} t} \int_{\mathbb{R}^{N}} \mathbb{E}(\rho(t)) \psi \mathrm{d} x=\int_{\mathbb{R}^{N}} \mathbb{E}(\rho(t))\left(\operatorname{div}\left(\lambda D^{T} \nabla \psi\right)-c \cdot \nabla \psi\right) \mathrm{d} x+\mathcal{O}\left(\tau \eta^{2}+\tau / \varepsilon+\tau /(\varepsilon \eta)+1 / \eta\right)+\frac{\mathrm{d}}{\mathrm{d} t} \mathcal{O}(\tau \eta+\tau)$,

where we used $\varepsilon \eta^{2} / \lambda=1$.

Consider a sequence of parameters which satisfy

$$
\left\{\begin{array}{l}
\tau_{n} \rightarrow 0, \quad \varepsilon_{n} \rightarrow 0, \quad \lambda_{n} \rightarrow \lambda_{\infty} \in[0, \infty), \quad \eta_{n} \rightarrow \infty \\
\frac{\varepsilon_{n} \eta_{n}^{2}}{\lambda_{n}}=1, \quad \frac{\tau_{n}}{\varepsilon_{n}} \rightarrow 0
\end{array}\right.
$$

Then by Corollary 1 , Lemma 5 and standard arguments we can exhibit $r \in \mathrm{C}^{0}\left([0, \infty) ; \mathcal{M}^{1}\left(\mathbb{R}^{N}\right)-\right.$ weak- $\left.-\star\right)$ and a subsequence such that, for any $0<T<\infty, \mathbb{E} \rho_{n} \rightarrow r$ in $\mathrm{C}^{0}\left([0, T] ; \mathcal{M}^{1}\left(\mathbb{R}^{N}\right)-\right.$ weak $\left.-\star\right)$. Then, passing to the limit in the above equation concludes the proof of Theorem 1 . Non negativity of the symmetric part of the diffusivity matrix $D$ is proved by repeating arguments in [23].

\subsection{Proof of Theorems 2 and 3}

Multiplying (2) by a test function $\psi \in \mathrm{C}_{c}^{\infty}\left(\mathbb{R}^{2 N}\right)$ and integrating yields the following weak formulation

$$
\frac{\mathrm{d}}{\mathrm{d} t} \int_{\mathbb{R}^{N}} \int_{\mathbb{R}^{N}} \mathbb{E}(f(t)) \psi \mathrm{d} v \mathrm{~d} x-\int_{\mathbb{R}^{N}} \int_{\mathbb{R}^{N}} \mathbb{E}(f(t)) v \cdot\left(\nabla_{x}-\frac{1}{\tau} \nabla_{v}\right) \psi \mathrm{d} v \mathrm{~d} x=+\frac{\eta}{\tau} \mathbb{E} \int_{\mathbb{R}^{N}} \int_{\mathbb{R}^{N}} f(t) u(t) \cdot \nabla_{v} \psi \mathrm{d} v \mathrm{~d} x .
$$

All the difficulties are thus concentrated in the right hand side; in particular, since in the scaling assumptions $\eta \rightarrow \infty$, it is not obvious at all that this integral remains bounded uniformly with respect to the parameters. As in the previous Section, we follow the method of [23] which uses the Duhamel formula to write $f(t)$ by means of $f(t-\varepsilon)$ so that the singular term can be cancelled thanks to the independency property of Lemma 1 . Since the proof is very close to [23], we only sketch the main arguments. As in the previous section, $\mathcal{O}(\beta)$ stands for a time dependent function, possibly random, which is bounded by $C \beta$ where $C$ does not depend on time, on the random variable nor on the parameters $\varepsilon, \eta, \lambda, \tau$. 
Let us introduce the group of operators $S_{t}(\Phi)(x, v)=\Phi(x-v t, v), t \in \mathbb{R}$. We note that:

- $S_{t}$ is unitary on $\mathrm{L}^{\infty}\left(\mathbb{R}^{2 N}\right)$;

- its formal adjoint is $S_{-t}$;

- the commutator formula $\nabla_{v} S_{t}=S_{t}\left(\nabla_{v}-t \nabla_{x}\right)$ holds.

Hence, we are led to

$$
\begin{aligned}
\int_{\mathbb{R}^{N}} \int_{\mathbb{R}^{N}} f(t) \phi \mathrm{d} v \mathrm{~d} x= & \int_{\mathbb{R}^{N}} \int_{\mathbb{R}^{N}} f(t-\varepsilon s) S_{-\varepsilon s} \phi \mathrm{d} v \mathrm{~d} x \\
& +\frac{\varepsilon}{\tau} \int_{0}^{s} \int_{\mathbb{R}^{N}} \int_{\mathbb{R}^{N}} f(t-\varepsilon \sigma) \eta u(t-\varepsilon \sigma) \cdot S_{-\varepsilon \sigma}\left(\nabla_{v}+\varepsilon \sigma \nabla_{x}\right) \phi \mathrm{d} v \mathrm{~d} x \mathrm{~d} \sigma \\
& -\frac{\varepsilon}{\tau} \int_{0}^{s} \int_{\mathbb{R}^{N}} \int_{\mathbb{R}^{N}} f(t-\varepsilon \sigma) v \cdot S_{-\varepsilon \sigma}\left(\nabla_{v}+\varepsilon \sigma \nabla_{x}\right) \phi \mathrm{d} v \mathrm{~d} x \mathrm{~d} \sigma,
\end{aligned}
$$

for any (smooth enough) test function $\phi$, random or not, and $t \geq \varepsilon, s \in[0,1]$.

\subsubsection{Proof of Theorem 3}

Throughout this section, we set $\tau=1$. First, we use $(23)$ with $\phi=\eta u(t) \cdot \nabla_{v} \psi$ and $s=1$. We note that $\left(\nabla_{v}+\varepsilon \sigma \nabla_{x}\right) \phi=\mathcal{O}(\eta)+(\eta \varepsilon / \lambda)$. Therefore, by using Lemma 2, the second integral in the right hand side has order $\mathcal{O}\left(\varepsilon \eta^{2}\right)+\mathcal{O}\left(\varepsilon^{2} \eta^{2} / \lambda\right)$ while, since $\psi$ is compactly supported, the third integral is $\mathcal{O}(\varepsilon \eta)+\mathcal{O}\left(\varepsilon^{2} \eta / \lambda\right)$. This motivates the scaling announced in Section 2:

$$
\eta=\frac{1}{\sqrt{\varepsilon}}, \quad \frac{\varepsilon}{\lambda} \rightarrow \kappa_{\infty} \in[0, \infty)
$$

From now on, we consider coefficients verifying these relations.

Indeed, taking the expectation, using Lemma 1, assumption (9) and Corollary 1, we obtain, for $t \geq \varepsilon$,

$$
\frac{1}{\sqrt{\varepsilon}} \mathbb{E} \int_{\mathbb{R}^{N}} \int_{\mathbb{R}^{N}} f(t) u(t) \cdot \nabla_{v} \psi \mathrm{d} v \mathrm{~d} x=\mathbb{E} \int_{0}^{1} \int_{\mathbb{R}^{N}} \int_{\mathbb{R}^{N}} f(t-\varepsilon \sigma) \Phi(t, \sigma) \mathrm{d} v \mathrm{~d} x \mathrm{~d} \sigma+\mathcal{O}(\sqrt{\varepsilon})
$$

with

$$
\Phi(t, \sigma)=u(t-\varepsilon \sigma) \cdot S_{-\varepsilon \sigma}\left(\nabla_{v}+\varepsilon \sigma \nabla_{x}\right)\left(u(t) \cdot \nabla_{v} \psi\right) .
$$

Furthermore, arguing as in the previous section or in [23], we are then able to prove that the family of functions $\left\{\iint \mathbb{E} f(t) \psi \mathrm{d} x \mathrm{~d} v\right\}$ lies in a compact set of $\mathrm{C}^{0}([0, T])$. (We first consider the shifted functions $\iint \mathbb{E} f(t+\varepsilon) \psi \mathrm{d} x \mathrm{~d} v$ by coming back to (22) and using this estimate on the right hand side.) Accordingly, we can work with a subsequence verifying $\mathbb{E} f(t) \rightarrow F(t)$ in $\mathrm{C}^{0}\left([0, T] ; \mathcal{M}^{1}\left(\mathbb{R}^{2 N}\right)-\right.$ weak $\left.-\star\right)$ for any $T>0$.

It remains to compute the limit of the leading term in (24). Using $(23)$, we have for $\sigma \in[0,1]$,

$$
\begin{aligned}
\mathbb{E} \int_{\mathbb{R}^{N}} \int_{\mathbb{R}^{N}} f(t-\varepsilon \sigma) \Phi \mathrm{d} v \mathrm{~d} x= & \mathbb{E} \int_{\mathbb{R}^{N}} \int_{\mathbb{R}^{N}}(f-\mathbb{E} f)(t-2 \varepsilon) S_{-(2-\sigma) \varepsilon} \Phi \mathrm{d} v \mathrm{~d} x \\
& +\mathbb{E} \int_{\mathbb{R}^{N}} \int_{\mathbb{R}^{N}}\left(f(t-2 \varepsilon)-f(t) S_{2 \varepsilon}\right) S_{-(2-\sigma) \varepsilon} \mathbb{E} \Phi \mathrm{d} v \mathrm{~d} x \\
& +\int_{\mathbb{R}^{N}} \int_{\mathbb{R}^{N}} \mathbb{E}(f(t)) S_{\sigma \varepsilon} \mathbb{E} \Phi \mathrm{d} v \mathrm{~d} x+\mathcal{O}\left(\sqrt{\varepsilon} \times\left(\left\|\nabla_{v} \Phi\right\|_{\infty}+\varepsilon\left\|\nabla_{x} \Phi\right\|_{\infty}\right)\right) .
\end{aligned}
$$

Since $\nabla_{v} \Phi$ and $\varepsilon \nabla_{x} \Phi$ are $\mathcal{O}(1)$, we realize that the remainder is actually $\mathcal{O}(\sqrt{\varepsilon})$. Furthermore, combining this bound on $\left(\nabla_{v}+\varepsilon \nabla_{x}\right) \Phi$ to the estimates of Lemma 2 and coming back to (23), the second integral of the right hand is also shown to be $\mathcal{O}(\sqrt{\varepsilon})$. Eventually, due to the independence property of Lemma 1 , the first term of 
the right hand side vanishes for any $t \geq 2 \varepsilon$. Accordingly, equation (24) becomes

$$
\frac{1}{\sqrt{\varepsilon}} \mathbb{E} \int_{\mathbb{R}^{N}} \int_{\mathbb{R}^{N}} f(t) u(t) \cdot \nabla_{v} \psi \mathrm{d} v \mathrm{~d} x=\int_{\mathbb{R}^{N}} \int_{\mathbb{R}^{N}} \mathbb{E}(f(t))\left(\int_{0}^{1} S_{\sigma \varepsilon} \mathbb{E} \Phi(t, \sigma) \mathrm{d} \sigma\right) \mathrm{d} v \mathrm{~d} x+\mathcal{O}(\sqrt{\varepsilon}) .
$$

It remains to compute $\mathbb{E} \Phi$. We have, by using (8),

$$
\begin{aligned}
\mathbb{E} \Phi(t, \sigma)= & \mathbb{E}\left(u(t-\varepsilon \sigma) \cdot S_{-\varepsilon \sigma}\left(\nabla_{v}+\varepsilon \sigma \nabla_{x}\right)\left(u(t) \cdot \nabla_{v} \psi\right)\right) \\
= & \sum_{i, j=1}^{N} \mathbb{E}\left(U_{i}\left(\frac{t}{\varepsilon}-\sigma, \frac{x}{\lambda}\right) U_{j}\left(\frac{t}{\varepsilon}, \frac{x}{\lambda}+\frac{\varepsilon}{\lambda} \sigma v\right)\right) \partial_{v_{i}} \partial_{v_{j}} \psi(x, v) \\
& +\sum_{i, j=1}^{N} \mathbb{E}\left(U_{i}\left(\frac{t}{\varepsilon}-\sigma, \frac{x}{\lambda}\right) \frac{\varepsilon}{\lambda} \sigma \partial_{x_{i}} U_{j}\left(\frac{t}{\varepsilon}, \frac{x}{\lambda}+\frac{\varepsilon}{\lambda} \sigma v\right)\right) \partial_{v_{j}} \psi(x, v) \\
& +\mathcal{O}(\varepsilon) .
\end{aligned}
$$

(The term $\mathcal{O}(\varepsilon)$ takes into account the difference between $\psi(x+\varepsilon \sigma v, v)$ and $\psi(x, v)$ and terms involving cross derivatives $\varepsilon \partial_{x_{i}} \partial_{v_{j}}$ of the test function.) Using (8) again and (9) yields

$$
\begin{aligned}
\mathbb{E} \Phi(t, \sigma)= & \sum_{i, j=1}^{N} R_{j, i}\left(\sigma, \frac{\varepsilon}{\lambda} \sigma v\right) \partial_{v_{i}} \partial_{v_{j}} \psi(x, v) \\
& +\sum_{i, j=1}^{N} \frac{\varepsilon}{\lambda} \sigma \partial_{x_{i}} R_{j, i}\left(\sigma, \frac{\varepsilon}{\lambda} \sigma v\right) \partial_{v_{j}} \psi(x, v)+\mathcal{O}(\varepsilon) \\
= & \sum_{i, j=1}^{N} \partial_{v_{i}}\left(R_{j, i}\left(\sigma, \kappa_{\infty} \sigma v\right) \partial_{v_{j}} \psi(x, v)\right)+\mathcal{O}(\varepsilon)+\mathcal{O}\left(\frac{\varepsilon}{\lambda}-\kappa_{\infty}\right) .
\end{aligned}
$$

Inserting this formula in (25) we can pass to the limit in (22) which ends the proof.

\subsubsection{Proof of Theorem 2}

The proof starts by coming back to Lemma 4 which shows that the crucial term to perform the hydrodynamic limit, provided $\tau \eta^{2} \rightarrow 0$, is

$$
\int_{\mathbb{R}^{N}} \rho(t) \eta u(t) \cdot \nabla_{x} \psi \mathrm{d} x=\int_{\mathbb{R}^{N}} \int_{\mathbb{R}^{N}} f(t) \eta u(t) \cdot \nabla_{x} \psi \mathrm{d} v \mathrm{~d} x,
$$

$\psi$ being a smooth enough test function depending only on the space variable. Therefore, let us use (23) with $\phi=\eta u(t) \cdot \nabla_{x} \psi$. Notice that $\left(\nabla_{v}+\varepsilon \sigma \nabla_{x}\right) \phi=\varepsilon \sigma \nabla_{x} \phi=\mathcal{O}(\varepsilon \eta / \lambda)+\mathcal{O}(\varepsilon \eta)$, so that, by using Lemma 2 , the two last integrals in the right hand side of $(23)$ are $\mathcal{O}\left(\frac{\varepsilon}{\tau} \times \eta \times \frac{\varepsilon \eta}{\lambda}\right)$. It motivates to set

$$
\varepsilon^{2} \eta^{2}=\tau \lambda
$$

Consequently, combining Lemma 4 to (23), Lemma 1 and (9), and reasoning as in the proof of Corollary 2, we are able to prove the compactness of the family $\left\{\mathbb{E} \int \rho(t) \psi \mathrm{d} x\right\}$ in $\mathrm{C}^{0}([0, T])$. Thus, we can exhibit a subsequence such that $\mathbb{E} \rho$ converges to some $r$ in $\mathrm{C}^{0}\left([0, T], \mathcal{M}^{1}\left(\mathbb{R}^{N}\right)-\right.$ weak $\left.-\star\right)$. Hence, we are left with the task of computing the expectation of (26). Let us set

$$
\Phi(t, \sigma)=\frac{\varepsilon \eta^{2}}{\tau}\left(u(t-\varepsilon \sigma)-\frac{1}{\eta} v\right) \cdot S_{-\varepsilon \sigma} \varepsilon \sigma \nabla_{x}\left(u(t) \cdot \nabla_{x} \psi\right) .
$$


Repeating the tricks of the previous section, we can rewrite $\mathbb{E}(26)$ as follows

$$
\begin{aligned}
\mathbb{E} \int_{\mathbb{R}^{N}} \int_{\mathbb{R}^{N}} f(t) \eta u(t) \cdot \nabla_{x} \psi \mathrm{d} v \mathrm{~d} x & =\mathbb{E} \int_{0}^{1} \int_{\mathbb{R}^{N}} \int_{\mathbb{R}^{N}} f(t-\varepsilon \sigma) \Phi \mathrm{d} v \mathrm{~d} x \mathrm{~d} \sigma \\
& =\int_{0}^{1} \int_{\mathbb{R}^{N}} \int_{\mathbb{R}^{N}} \mathbb{E} f(t) S_{\sigma \varepsilon} \mathbb{E} \Phi \mathrm{d} v \mathrm{~d} x \mathrm{~d} \sigma+\mathcal{O}(1 / \eta) .
\end{aligned}
$$

By using (8) and (9), we get

$$
\begin{aligned}
\mathbb{E} \Phi(t, \sigma)= & \frac{\varepsilon \eta^{2}}{\tau} \mathbb{E}\left(\left(u(t-\varepsilon \sigma)-\frac{1}{\eta} v\right) \cdot S_{-\varepsilon \sigma} \varepsilon \sigma \nabla_{x}\left(u(t) \cdot \nabla_{x} \psi\right)\right) \\
= & \frac{\varepsilon^{2} \eta^{2}}{\tau} \sigma \sum_{i, j=1}^{N} \mathbb{E}\left\{\left(U_{i}\left(\frac{t}{\varepsilon}-\sigma, \frac{x}{\lambda}\right)-\frac{v_{i}}{\eta}\right)\right. \\
& \left.\times\left(\frac{1}{\lambda} \partial_{x_{i}} U_{j}\left(\frac{t}{\varepsilon}, \frac{x}{\lambda}+\frac{\varepsilon}{\lambda} \sigma v\right) \partial_{x_{j}} \psi(x)+U_{j}\left(\frac{t}{\varepsilon}, \frac{x}{\lambda}+\frac{\varepsilon}{\lambda} \sigma v\right) \partial_{x_{i} x_{j}}^{2} \psi(x)\right)\right\}+\mathcal{O}(\varepsilon) \\
= & \frac{\varepsilon^{2} \eta^{2}}{\tau \lambda} \sigma \sum_{i, j=1}^{N} \partial_{x_{i}} R_{j, i}\left(\sigma, \frac{\varepsilon}{\lambda} \sigma v\right) \partial_{x_{j}} \psi(x) \\
& +\frac{\varepsilon^{2} \eta^{2}}{\tau} \sigma \sum_{i, j=1}^{N} R_{j, i}\left(\sigma, \frac{\varepsilon}{\lambda} \sigma v\right) \partial_{x_{i}} \partial_{x_{j}} \psi(x, v)+\mathcal{O}(\varepsilon) .
\end{aligned}
$$

Plugging this formula in (27), and recalling from (13) that $\varepsilon / \lambda \rightarrow 0, \varepsilon^{2} \eta^{2} /(\tau \lambda)=1, \lambda \rightarrow 0$ and $\eta \rightarrow \infty$ allow us to pass to the limit and ends the proof of Theorem 2.

\section{REFERENCES}

[1] P. Berthonnaud, Limites fluides pour des modèles cinétiques de brouillards de gouttes monodispersés. C. R. Acad. Sci. 331 (2000) 651-654.

[2] M. Brassart, Limite semi-classique de transformées de Wigner dans des milieux périodiques ou aléatoires. Thèse Université de Nice-Sophia Antipolis (Novembre 2002).

[3] J.R. Brock and G.M. Hidy, The dynamics of aerocolloidal systems. Pergamon Press (1970).

[4] R. Caflisch and G. Papanicolaou, Dynamic theory of suspensions with Brownian effects. SIAM J. Appl. Math. 43 (1983) $885-906$.

[5] J.F. Clouet and K. Domelevo, Solutions of a kinetic stochastic equation modeling a spray in a turbulent gas flow. Math. Models Methods Appl. Sci. 7 (1997) 239-263.

[6] L. Desvillettes, About the modeling of complex flows by gas-particles methods, Proceedings of the workshop "Trends in Numerical and Physical Modeling for Industrial Multiphase Flows", Cargèse, France (2000).

[7] K. Domelevo and M.-H. Vignal, Limites visqueuses pour des systèmes de type Fokker-Planck-Burgers unidimensionnels. $C$. $R$. Acad. Sci. 332 (2001) 863-868.

[8] K. Domelevo and P. Villedieu, Work in preparation. Personal communication.

[9] S. Gavrilyuck and V. Teshukhov, Kinetic model for the motion of compressible bubbles in a perfect fluid. Eur. J. Mech. B/Fluids 21 (2002) 469-491.

[10] F. Golse, in From kinetic to macroscopic models in Kinetic equations and asymptotic theory, B. Perthame and L. Desvillettes Eds., Gauthier-Villars, Ser. Appl. Math. 4 (2000) 41-121.

[11] T. Goudon, Asymptotic problems for a kinetic model of two-phase flow. Proc. Royal Soc. Edimburgh 131 (2001) 1371-1384.

[12] T. Goudon, P.-E. Jabin and A. Vasseur, Hydrodymamic limit for the Vlasov-Navier-Stokes system: Light particles regime. Preprint.

[13] T. Goudon, P.-E. Jabin and A. Vasseur, Hydrodymamic limit for the Vlasov-Navier-Stokes system: Fine particles regime. Preprint.

[14] K. Hamdache, Global existence and large time behaviour of solutions for the Vlasov-Stokes equations. Japan J. Ind. Appl. Math. 15 (1998) 51-74. 
[15] H. Herrero, B. Lucquin-Desreux and B. Perthame, On the motion of dispersed balls in a potential flow: a kinetic description of the added mass effect. SIAM J. Appl. Math. 60 (1999) 61-83.

[16] P.-E. Jabin, Large time concentrations for solutions to kinetic equations with energy dissipation. Comm. Partial Differential Equations 25 (2000) 541-557.

[17] P.-E. Jabin, Macroscopic limit of Vlasov type equations with friction. Ann. IHP Anal. Non Linéaire 17 (2000) $651-672$.

[18] P.-E. Jabin and B. Perthame, in Notes on mathematical problems on the dynamics of dispersed particles interacting through a fluid in Modeling in applied sciences, a kinetic theory approach, N. Bellomo and M. Pulvirenti Eds., Birkhäuser (2000) $111-147$.

[19] P. Kramer and A. Majda, Simplified models for turbulent diffusion: Theory, numerical modeling, and physical phenomena. Physics Reports 314 (1999) 237-574.

[20] R. Kubo, Stochastic Liouville equations. J. Math. Phys. 4 (1963) 174-183.

[21] G. Loeper and A. Vasseur, Electric turbulence in a plasma subject to a strong magnetic field. Preprint.

[22] P.J. O'Rourke, Statistical properties and numerical implementation of a model for droplets dispersion in a turbulent gas. J. Comp. Phys. 83 (1989) 345-360.

[23] F. Poupaud and A. Vasseur, Classical and quantum transport in random media. J. Math. Pures Appl. 82 (2003) 711-748.

[24] G. Russo and P. Smereka, Kinetic theory for bubbly flows I, II. SIAM J. Appl. Math. 56 (1996) 327-371.

[25] C. Villani, A review of mathematical topics in collisional kinetic theory, in Handbook of mathematical fluid mechanics, S. Friedlander and D. Serre Eds., North-Holland (2002).

[26] F.A. Williams, Combustion theory. Benjamin Cummings Publ., 2nd edn. (1985).

[27] L.I. Zaichik, A statistical model of particle transport and heat transfer in turbulent shear flows. Phys. Fluids 11 (1999) $1521-1534$

To access this journal online: www.edpsciences.org 